\title{
REVISED Three datasets for nutrition environment measures of food outlets located in the Lower Mississippi Delta region of the United States [version 2; peer review: 2 approved]
}

Jessica L. Thomson (iD) , Alicia S. Landry²

${ }^{1}$ Delta Human Nutrition Research Program, USDA Agricultural Research Service, Stoneville, MS, 38776, USA

${ }^{2}$ Department of Family and Consumer Sciences, University of Central Arkansas, Conway, AR, 72035, USA

\section{V2 First published: 09 Nov 2020, 9:1307 \\ https://doi.org/10.12688/f1000research.27192.1}

Latest published: 24 May 2021, 9:1307

https://doi.org/10.12688/f1000research.27192.2

\section{Abstract}

This data note provides details of a research database containing 266 food outlets located in five rural towns in the Lower Mississippi Delta region of Mississippi, whose nutrition environments were measured from 2016 to 2018. The food outlet types include grocery stores, convenience stores, full-service restaurants, and fast food restaurants. The purpose of this publication is to describe the three datasets for external researchers who may be interested in making use of them. The datasets are available from the USDA National Agricultural Library's Ag Data Commons under a CCO 1.0 Universal License: https://doi.org/10.15482/USDA.ADC/1503704.

\section{Keywords}

Food environment, nutrition, grocery stores, convenience stores, restaurants, Nutrition Environment Measures Survey, Lower Mississippi Delta, rural

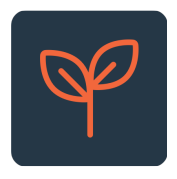

This article is included in the Agriculture, Food and Nutrition gateway.

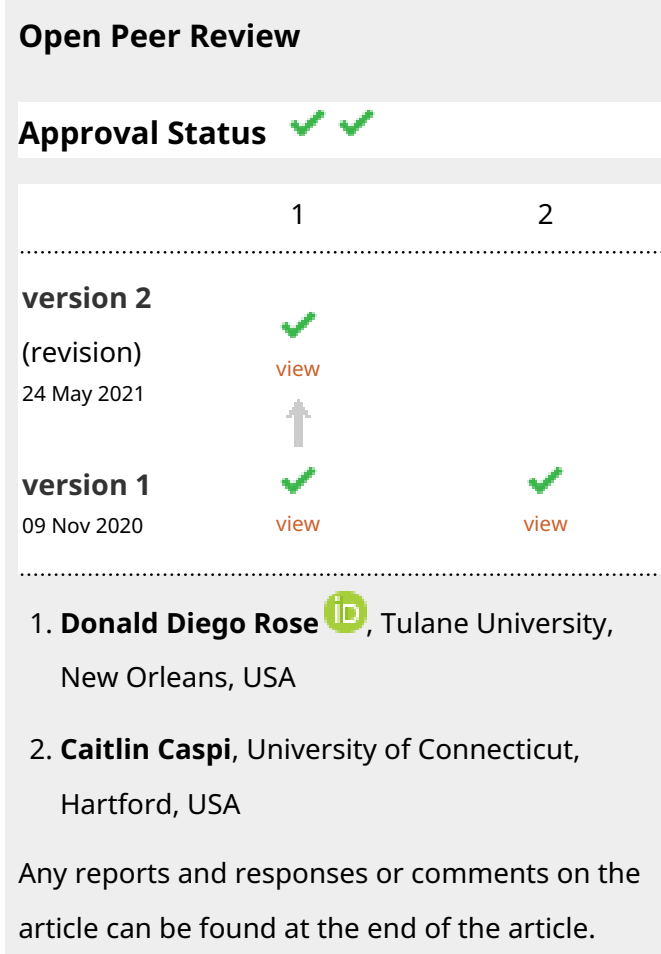


Corresponding author: Jessica L. Thomson (jessica.thomson@usda.gov)

Author roles: Thomson JL: Conceptualization, Data Curation, Formal Analysis, Funding Acquisition, Project Administration, Supervision, Writing - Original Draft Preparation, Writing - Review \& Editing; Landry AS: Conceptualization, Methodology, Supervision, Writing Review \& Editing

Competing interests: No competing interests were disclosed.

Grant information: This work was supported by the USDA Agricultural Research Service (Project 6001-51000-004-00D). The findings and conclusions in this publication are those of the authors and should not be construed to represent any official USDA or US government determination or policy. USDA is an equal opportunity provider and employer.

The funders had no role in study design, data collection and analysis, decision to publish, or preparation of the manuscript.

Copyright: $\odot 2021$ Thomson JL and Landry AS. This is an open access article distributed under the terms of the Creative Commons Attribution License, which permits unrestricted use, distribution, and reproduction in any medium, provided the original work is properly cited.

How to cite this article: Thomson JL and Landry AS. Three datasets for nutrition environment measures of food outlets located in the Lower Mississippi Delta region of the United States [version 2; peer review: 2 approved] F1000Research 2021, 9:1307 https://doi.org/10.12688/f1000research.27192.2

First published: 09 Nov 2020, 9:1307 https://doi.org/10.12688/f1000research.27192.1 


\section{REVISED Amendments from Version 1}

Based on two thoughtful and thorough reviews of this data note, the following changes to the text were made. (1) Population characteristics of the five towns and the researchers' reason for selecting the five towns were added. (2) Classification definitions for the food outlets - grocery stores, convenience stores, fullservice restaurants, and fast food restaurants - were added. (3) Clarification that all outlets were physically visited to ensure that they were open, sold food, and classified correctly was provided. (4) Expanded descriptions of the survey tools used to measure the food outlets were provided. (5) Clarification about discussion and resolution of measurement discrepancies was provided. (6) Clarification about the types of scores available in the datasets (raw scores, component scores, and total scores) was provided and mention of ratio scores was removed because they are not included in the datasets. (7) The US Department of Agriculture Food Environment Atlas reference was updated to directly link to the documentation.

Any further responses from the reviewers can be found at the end of the article

\section{Introduction}

The Mississippi River Delta region is among the most socioeconomically disadvantaged areas of the United States (US) with less healthful food environments (e.g., low access to healthful foods, food insecurity) and poorer health outcomes than non-Delta counties in the same states and the nation ${ }^{1}$. Accessibility (location of healthful food outlets near neighborhoods, particularly in low-income and rural areas), availability (healthful options in local food outlets), and affordability (reasonable prices) of nutrient-dense foods are crucial to facilitate adoption of a healthful $\operatorname{diet}^{2-4}$. To inform future nutrition interventions designed for residents of the Lower Mississippi, the Delta Food Outlets Study was conducted to measure nutrition environments of towns located in this region.

\section{Methods}

Delta Food Outlets was an observational study designed to collect data on food outlets located in five rural Lower Mississippi Delta towns. These towns were selected because participants of a previously conducted nutrition intervention resided in the five towns and assessing environmental exposures potentially influencing their dietary habits was of interest to researchers ${ }^{5}$. The population of the five towns ranged from 1,750 to 32,612 residents. The percentage of the towns'population that was African American ranged from $49 \%$ to $91 \%$ and the percentage that lived below the federal poverty level ranged from $29 \%$ to $51 \%$. Food outlet types included grocery stores, convenience stores, full-service restaurants, and fast food restaurants. The study was approved and classified as exempt by the Institutional Review Board of Delta State University. Data collection occurred from March 2016 through September 2018.

Grocery stores were identified by referencing two sources - the US Department of Agriculture (USDA) Food and Nutrition Service Supplemental Nutrition Assistance Program (SNAP) retailer locator ${ }^{6}$ and the Mississippi State Department of Health
Restaurant and Food Facility Inspections website ${ }^{7}$. Convenience stores were identified by referencing three sources - the SNAP retailer locator ${ }^{6}$, the B2B Yellow Pages website ${ }^{8}$, and lists of current privilege licenses obtained from city clerks. Restaurants were identified by referencing the Mississippi State Department of Health Restaurant and Food Facility Inspections website $^{7}$. Food outlets were classified using operational definitions contained in the Economic Research Service's Food Environment Atlas documentation'. Grocery stores were defined as supermarkets and smaller grocery stores primarily engaged in retailing a general line of food, such as canned and frozen foods; fresh fruits and vegetables; and fresh and prepared meats, fish, and poultry. Convenience stores were defined as stores primarily engaged in retailing a limited line of goods that generally includes milk, bread, soda, and snacks. Full-service restaurants were defined as restaurants that provide food service to patrons who order and are served while seated and pay after eating. Fast food restaurants were defined as restaurants that provide food services (excluding snack and nonalcoholic beverage bars) where patrons generally order or select items and pay before eating. Before measurement, all outlets were physically visited to ensure that they were open, sold food, and were classified correctly. While the 266 food outlets included in the datasets represent the entire population of these types of food outlets in the five towns, they may not be representative of all such outlets located in rural Lower Mississippi Delta towns.

Nutrition environments of the food outlets were measured using the Nutrition Environment Measures Survey (NEMS) for grocery stores (NEMS-S), convenience stores (NEMS-CS), and restaurants $(\mathrm{NEMS}-\mathrm{R})^{10}$. NEMS tools are validated observational measures of retail store nutrition environments that focus on the availability of healthful food choices, quality of fresh produce (acceptable or unacceptable based on visual inspection), and comparative pricing between healthful and less healthful options (e.g., lower vs. higher fat, no added vs. added sugars, whole grain vs. refined grain) in 11 common categories ${ }^{11}$. The categories included milk, fruits, vegetables, ground beef, hot dogs, frozen dinners, baked goods, beverages, bread, chips, and cereal. For the restaurants, main dishes and main dish salads were measured rather than ground beef, hot dogs, and frozen dinners, and baked goods and cereal were not measured. Additionally, facilitators (e.g., nutrition information available) and barriers (e.g., supersizing available) for healthful eating were measured in restaurants. A comprehensive description of the Delta Food Outlets Study methodology and measures has been published elsewhere ${ }^{5}$.

The NEMS tools were recreated as electronic surveys using Snap Surveys software (version 11.20, Snap Surveys Ltd). All data were collected via tablets loaded with Snap Surveys software and stored on the Snap WebHost, an online mobile and secure survey management system. For quality assurance purposes, $25 \%$ of the food outlets were randomly selected for duplicate measurement. Discrepancies between measurements were discussed between senior researchers and data collectors and resolved (e.g., outlet re-visited to determine correct value). 
Food outlets were scored using algorithms provided for the NEMS tools. Higher scores indicate a more healthful nutrition environment. The datasets include raw scores for each item, component scores (i.e., food groups, availability, pricing, quality, healthy options, facilitators, and barriers), and total scores. Scoring was performed using SAS® (version 9.4, SAS Institute Inc).

\section{Data availability}

USDA National Agricultural Library's Ag Data Commons: Delta Food Outlets Study, https://doi.org/10.15482/USDA.ADC/ $1503704^{12}$.
This project contains all three datasets - NEMS-C (convenience stores), NEMS-G (grocery stores), and NEMS-R (restaurants) - along with their corresponding data dictionaries.

Data are available under the terms of the Creative Commons Zero "No rights reserved" data waiver (CCO 1.0 Public domain dedication).

\section{Acknowledgements}

The authors are grateful to Melissa Goodman, Debra Johnson, and Donna Ransome for their research support and Delta Health Alliance for their in-kind support.
1. Gennuso KP, Jovaag A, Catlin BB, et al.: Assessment of Factors Contributing to Health Outcomes in the Eight States of the Mississippi Delta Region. Prev Chronic Dis. 2016; 13(3): E33.

PubMed Abstract | Publisher Full Text | Free Full Text

2. Cohen B, Andrews M, Kantor LS: Community Food Security Assessment Toolkit. 2002.

Reference Source

3. Laraia B, Siega-Riz AM, Kaufman JS, et al.: Proximity of supermarkets is positively associated with diet quality index for pregnancy. Prev Med. 2004 39(5): 869-875.

PubMed Abstract | Publisher Full Text

4. Rose D, Richards R: Food store access and household fruit and vegetable use among participants in the US Food Stamp Program. Public Health Nutr. 2004; 7(8): 1081-1088.

PubMed Abstract | Publisher Full Text

5. Thomson JL, Goodman MH, Landry AS: Measurement of Nutrition Environments in Grocery Stores, Convenience Stores, and Restaurants in the Lower Mississippi Delta. Prev Chronic Dis. 2020; 17: E24. PubMed Abstract | Publisher Full Text | Free Full Text

6. US Department of Agriculture Food and Nutrition Service: Where can I use
SNAP EBT? Accessed March 15, 2016. Reference Source

7. Mississippi State Department of Health: Restaurant and Food Facility Inspections. Accessed March 15, 2016. Reference Source

8. b2bBiz.com: B2BYellowpages.com. Accessed March 15, 2016 Reference Source

9. US Department of Agriculture Economic Research Service: Food Environment Atlas. Accessed August 8, 2019. Reference Source

10. The University of Pennsylvania: Nutrition Environment Measures Survey. Accessed May 23, 2018. Reference Source

11. Glanz K, Sallis JF, Saelens BE, et al.: Nutrition Environment Measures Survey in stores (NEMS-S): development and evaluation. Am J Prev Med. 2007; 32(4): 282-289.

PubMed Abstract | Publisher Full Text

12. Thomson JL: Delta Food Outlets Study. Ag Data Commons. Accessed 2020-10-19. http://www.doi.org/10.15482/USDA.ADC/1503704 


\section{Open Peer Review}

\section{Current Peer Review Status:}

\section{Version 2}

Reviewer Report 24 May 2021

https://doi.org/10.5256/f1000research.56999.r85979

(C) 2021 Rose D. This is an open access peer review report distributed under the terms of the Creative Commons Attribution License, which permits unrestricted use, distribution, and reproduction in any medium, provided the original work is properly cited.

\section{Donald Diego Rose}

School of Public Health \& Tropical Medicine, Tulane University, New Orleans, LA, USA

All looks good. No further comments.

Competing Interests: No competing interests were disclosed.

Reviewer Expertise: Public health nutrition, food security, food environment

I confirm that I have read this submission and believe that I have an appropriate level of expertise to confirm that it is of an acceptable scientific standard.

\section{Version 1}

Reviewer Report 17 May 2021

\section{https://doi.org/10.5256/f1000research.30043.r83927}

(C) 2021 Caspi C. This is an open access peer review report distributed under the terms of the Creative Commons Attribution License, which permits unrestricted use, distribution, and reproduction in any medium, provided the original work is properly cited.

\section{Caitlin Caspi}

Rudd Center for Food Policy and Obesity, University of Connecticut, Hartford, CT, USA

This a useful, detailed, and fairly recent data set that can be used by researchers to study the food environment in the Lower Mississippi Delta region. The data note concisely describes the data and is likely to be helpful documentation. 
There were some places where more detail would have been useful.

1. What are the characteristics of the towns of interest? Adding in the range of population size of the towns would be helpful. Were there large metropolitan areas that were excluded because this study was intentionally focused on towns? And/or can the authors make any statement in the note about how the towns may not be representative of the larger Lower Mississippi Delta area food environment? Some information like this would be helpful for readers in determining if the dataset is appropriate for use for addressing their research question.

2. Classifying food retail outlets (i.e., grocery stores vs. convenience stores, restaurants vs. fast food outlets) can be challenging. Potential misclassification of store types is a major topic of discussion in food environment databases. Consider adding in more detail about how food stores were classified. The definitions used for the food retail outlet categories would be helpful (I see they are defined in the longer paper), but even more helpful would be a description of how decisions were made about how to classify a food outlet (e.g., based on one of the food lists, and then verified during data collection in person?).

3. Relatedly, the authors reference the documentation for the USDA Food Environment Atlas for how stores were classified. However, the link goes to the Atlas, not the documentation, so it's hard to find more information on how stores were classified. It would be helpful to have a direct link.

Is the rationale for creating the dataset(s) clearly described?

Yes

Are the protocols appropriate and is the work technically sound? Yes

Are sufficient details of methods and materials provided to allow replication by others? Partly

Are the datasets clearly presented in a useable and accessible format?

Yes

Competing Interests: No competing interests were disclosed.

Reviewer Expertise: Food environment, food security, policies and interventions to address dietrelated behaviors

I confirm that I have read this submission and believe that I have an appropriate level of expertise to confirm that it is of an acceptable scientific standard.

Author Response 18 May 2021

Jessica Thomson, USDA Agricultural Research Service, Stoneville, USA

We thank Dr. Caspi for their thoughtful and thorough review of our manuscript. We have 
incorporated Dr. Caspi's suggestions for strengthening our Data Note about the Delta Food Outlets datasets. Our responses to Dr. Caspi's comments follow.

Pertaining to the towns: What are the characteristics of the towns of interest? Adding in the range of population size of the towns would be helpful. Were there large metropolitan areas that were excluded because this study was intentionally focused on towns? And/or can the authors make any statement in the note about how the towns may not be representative of the larger Lower Mississippi Delta area food environment? Some information like this would be helpful for readers in determining if the dataset is appropriate for use for addressing their research question.

Response: We have added characteristics of the 5 towns including ranges for population size, percentage of African American residents, and percentage of residents living below the federal poverty level. Additionally, we added our reason for selecting these 5 towns - because participants of a previously conducted nutrition intervention resided in the 5 towns and assessing environmental exposures potentially influencing their dietary habits was of interest. We chose not to add a note about the non-representative nature of the towns because we felt it was redundant with our comment about the non-representative nature of the food outlets.

Classifying food retail outlets (i.e., grocery stores vs. convenience stores, restaurants vs. fast food outlets) can be challenging. Potential misclassification of store types is a major topic of discussion in food environment databases. Consider adding in more detail about how food stores were classified. The definitions used for the food retail outlet categories would be helpful (I see they are defined in the longer paper), but even more helpful would be a description of how decisions were made about how to classify a food outlet (e.g., based on one of the food lists, and then verified during data collection in person?). Response: We have added more detail about definitions used to classify food outlets. Additionally, we added that before measurement, all outlets were physically visited to ensure that they were open, sold food, and were classified correctly.

Relatedly, the authors reference the documentation for the USDA Food Environment Atlas for how stores were classified. However, the link goes to the Atlas, not the documentation, so it's hard to find more information on how stores were classified. It would be helpful to have a direct link.

Response: We have updated the Food Environment Atlas reference to include a direct link to the documentation.

Competing Interests: The authors have no competing interests to disclose.

Reviewer Report 22 December 2020

https://doi.org/10.5256/f1000research.30043.r74475

(c) 2020 Rose D. This is an open access peer review report distributed under the terms of the Creative Commons Attribution License, which permits unrestricted use, distribution, and reproduction in any medium, provided the original work is properly cited. 


\section{Donald Diego Rose}

School of Public Health \& Tropical Medicine, Tulane University, New Orleans, LA, USA

Congratulations on a great piece of primary research on the food environment in the lower Mississippi Delta! I think it is great that you are listing the datasets in a way that can be found by other researchers, and the Data Note through F1000Resaerch is a great vehicle for this. I have just a few comments to strengthen the Note for potential users. See below for some minor changes to be made:

1. "located in five rural Lower Mississippi Delta towns of interest to researchers"

I think it would be useful to explain why or how these towns were chosen. Why were they of interest to the researchers? Are they representative of a specific region? Was it just a convenience sample? Do they provide a range of geographies or socio-economic conditions? I realize this is probably in the source article (\#5), but a couple of sentences here answering one or more of these questions to orient potential users of the data would be helpful.

Also, can you list the names of the towns? If there is a confidentiality issue, I would understand. But if not, it would be helpful for potential users of the data to know which towns.

2. "that focus on the availability of healthful food choices, quality of fresh produce, and comparative pricing between healthful and less healthful options in 11 common categories"

I know this is well documented in other places, but it would be useful in this Data Note to list one example of each of these and to also list all 11 food categories. You could add parenthetical comments, for example:

availability of healthful food choices (e.g, non-fat milk or whole grain bread, etc), quality of fresh produce (as scored on a X-point scale), and...11 common categories. The categories include $a, b$, c, ....

Then list them all. It's easy to do and gives the reader a better sense of the data.

3. "Discrepancies between measurements were discussed and resolved."

Discussed by whom? The investigators? Graduate student assistants? Just make clear this clear.

4. "The higher the ratio score, the more healthful the nutrition environment."

Can you indicate if the raw scores and the component scores are also available on the database? Either way is fine, but this would be helpful to know because some feel that the overall NEMS measures are like black box numbers that may not be helpful for a specific campaign to increase fruits and vegetables. See, for example, the references below that allow assessment for targeted campaigns to increase fruits and vegetables or decrease empty calorie snack foods. You may wish to include one or both of these references. 
Farley TA, Rice JC, Bodor JN, Cohen DA, Blumenthal RN, Rose D. "Measuring the Food Environment: Shelf Space of Fruits, Vegetables, and Snack Foods in Stores," Journal of Urban Health, 2009;86:672-682. ${ }^{1}$

Miller C, Bodor JN, Rose D. "Measuring the food environment: A systematic technique for characterizing food stores using display counts," Journal of Environmental and Public Health 2012; doi:10.1155/2012/707860.2

\section{References}

1. Farley TA, Rice J, Bodor JN, Cohen DA, et al.: Measuring the food environment: shelf space of fruits, vegetables, and snack foods in stores.J Urban Health. 2009; 86 (5): 672-82 PubMed Abstract | Publisher Full Text

2. Miller C, Bodor JN, Rose D: Measuring the food environment: a systematic technique for characterizing food stores using display counts.J Environ Public Health. 2012; 2012: 707860 PubMed Abstract | Publisher Full Text

Is the rationale for creating the dataset(s) clearly described?

Yes

Are the protocols appropriate and is the work technically sound?

Yes

Are sufficient details of methods and materials provided to allow replication by others? Partly

Are the datasets clearly presented in a useable and accessible format?

Partly

Competing Interests: No competing interests were disclosed.

Reviewer Expertise: Public health nutrition, food security, food environment

I confirm that I have read this submission and believe that I have an appropriate level of expertise to confirm that it is of an acceptable scientific standard.

Author Response 18 May 2021

Jessica Thomson, USDA Agricultural Research Service, Stoneville, USA

We thank Dr. Rose for their thoughtful and thorough review of our manuscript. We have incorporated Dr. Rose's suggestions for strengthening our Data Note about the Delta Food Outlets datasets. Our responses to Dr. Rose's comments follow.

Pertaining to the towns: I think it would be useful to explain why or how these towns were chosen. Why were they of interest to the researchers? Are they representative of a specific region? Was it just a convenience sample? Do they provide a range of geographies or socioeconomic conditions? I realize this is probably in the source article (\#5), but a couple of 
sentences here answering one or more of these questions to orient potential users of the data would be helpful. Also, can you list the names of the towns? If there is a confidentiality issue, I would understand. But if not, it would be helpful for potential users of the data to know which towns.

Response: We have added our reason for selecting these 5 towns - because participants of a previously conducted nutrition intervention resided in the 5 towns and assessing environmental exposures potentially influencing their dietary habits was of interest. We have chosen not to name the 5 towns due to confidentiality issues.

Pertaining to the NEMS instruments: I know this is well documented in other places, but it would be useful in this Data Note to list one example of each of these and to also list all 11 food categories. You could add parenthetical comments, for example: availability of healthful food choices (e.g, non-fat milk or whole grain bread, etc), quality of fresh produce (as scored on a $X$-point scale), and... 11 common categories. The categories include $a, b, c, .$. Then list them all. It's easy to do and gives the reader a better sense of the data.

Response: We have added examples of the measures' focus and listed the 11 common categories as suggested.

Pertaining to measurement discrepancies: Discussed by whom? The investigators? Graduate student assistants? Just make clear this clear.

Response: We have clarified that discrepancies were discussed between senior researchers and data collectors and resolved (e.g., outlet re-visited to determine correct value).

Pertaining to the scores: Can you indicate if the raw scores and the component scores are also available on the database? Either way is fine, but this would be helpful to know because some feel that the overall NEMS measures are like black box numbers that may not be helpful for a specific campaign to increase fruits and vegetables. See, for example, the references below that allow assessment for targeted campaigns to increase fruits and vegetables or decrease empty calorie snack foods. You may wish to include one or both of these references. Farley TA, Rice JC, Bodor JN, Cohen DA, Blumenthal RN, Rose D.

"Measuring the Food Environment: Shelf Space of Fruits, Vegetables, and Snack Foods in Stores," Journal of Urban Health, 2009;86:672-682.1. Miller C, Bodor JN, Rose D. "Measuring the food environment: A systematic technique for characterizing food stores using display counts," Journal of Environmental and Public Health 2012; doi:10.1155/2012/707860.

Response: We apologize for this oversight in describing our datasets. Raw scores, component scores, and total scores are available in the datasets. In fact, we did not include ratio scores in the datasets because they were created specifically for the purposes of our study and may not be relevant or useful for other researchers. Hence, we have added text about the availability of raw, component, and total scores and removed the text about ratio scores. While the references provided are very interesting, we did not feel it was necessary to include them in our Data Note given the availability of raw scores in the datasets.

Competing Interests: The authors have no competing interests to disclose. 
The benefits of publishing with F1000Research:

- Your article is published within days, with no editorial bias

- You can publish traditional articles, null/negative results, case reports, data notes and more

- The peer review process is transparent and collaborative

- Your article is indexed in PubMed after passing peer review

- Dedicated customer support at every stage

For pre-submission enquiries, contact research@f1000.com 